\title{
Application of novel nanotechnologies in asthma
}

\author{
Adriana Lopes da Silva ${ }^{1,2,3}$, Luisa Andrade Silva ${ }^{1,3}$, Fernanda Ferreira Cruz ${ }^{1,3}$, \\ Patricia Rieken Macedo Rocco ${ }^{1,3}$, Marcelo Marcos Morales ${ }^{2,3}$
}

${ }^{1}$ Laboratory of Pulmonary Investigation, ${ }^{2}$ Laboratory of Cellular and Molecular Biology, Federal University of Rio de Janeiro, Rio de Janeiro, Brazil; ${ }^{3}$ Rede NanoSaúde, Fundação Carlos Chagas Filho de Amparo a Pesquisa do Estado do Rio de Janeiro, Rio de Janeiro, Brazil

Correspondence to: Marcelo Marcos Morales. Universidade Federal do Rio de Janeiro, Centro de Ciências da Saúde, Instituto de Biofísica Carlos Chagas Filho, Avenida Carlos Chagas Filho 373, Bloco G - 014, Cidade Universitária, Rio de Janeiro - RJ, Brasil.

Email: marcelo.morales@mctic.gov.br.

Provenance and Peer Review: This article was commissioned by the Editorial Office, Annals of Translational Medicine. The article did not undergo external peer review.

Comment on: Wang L, Feng M, Li Q, et al. Advances in nanotechnology and asthma. Ann Transl Med 2019;7:180.

Submitted Oct 28, 2019. Accepted for publication Dec 04, 2019.

doi: $10.21037 / \mathrm{atm} .2019 .12 .40$

View this article at: http://dx.doi.org/10.21037/atm.2019.12.40

Asthma, is a very prevalent chronic pulmonary disease, affecting over 230 million people worldwide, with available treatments limited to symptoms relief (1). Considering this serious world situation, Wang et al. highlighted the nanotechnology related to delivery of drugs or biological materials, reducing side toxic effects, increasing drug bioavailability, thus improving asthma treatment. However, in this editorial comment, we summarize the challenges that need to be overcome to nanotherapy become pertinent in clinical practice.

This respiratory pathology is characterized by a chronic inflammation of the lower airways, triggered by environmental antigens, in the presence (atopic asthma) or absence (allergic asthma) of a genetic predisposition (2). In general, these immune responses lead to airway hyperreactivity, progressive extracellular matrix changes (i.e., lung collagen deposition), and airflow limitation $(3,4)$.

Despite advances in understanding the pathophysiology of asthma, improvements in diagnosis, and the introduction of new therapies-phosphodiesterase inhibitors, longacting bronchodilators, anti-IgE monoclonal antibody, and leukotriene inhibitors-asthma remains incurable and a major public health problem, without signs of declining prevalence (5-7). Although the first-line treatment of choice is corticosteroid therapy, alone or in combination with $\beta 2$-adrenergic agonists, these medications cannot modify disease progression. In Vol 7 No 8 of Annals of Translational Medicine, Wang et al. suggested that these treatments have limited efficacy due to the heterogeneity and complexity of asthma. Therefore, alternative therapeutic strategies are needed to mitigate both inflammatory and remodeling processes in different asthma phenotypes, improving lung function without leading to adverse events, such as immunosuppression.

In this scenario, Wang et al. highlighted how nanostructured materials can be used as nanocarriers, either of existing drugs traditionally used to relieve asthma symptoms (such as hormones and bronchodilators) or of brand-new drugs that can attenuate the inflammatory process and airway hyperresponsiveness. They have focused on studies with organic-based nanoparticulate systems, which are typically biodegradable and nontoxic. Such nanocarriers protect the drug cargo against degradation or inactivation after administration; facilitate its delivery and enhance its levels in target tissue; promote controlled release of their cargo, which avoids toxicity and leads to long-term beneficial effects; and provide stable expression of therapeutic mediators, while minimizing adverse effects $(8,9)$. In a recent report, for example, nanocarriermediated delivery of glucocorticoids led to 7 days of sustained anti-inflammatory effects in experimental asthma, enabling reduction of the dose and number of doses to be administered, which may be advantageous in clinical practice.

When designing a nanocarrier system for drug or gene therapies for asthma, however, some issues must be addressed. First, various physiological barriers-i.e., the mucus gel layer covering the lung airways, which is 
abnormally thickened in asthma, hindering widespread distribution of nanoparticles-make this therapeutic strategy very challenging $(10,11)$. Moreover, intratracheally delivered nanoparticles need to cross lung epithelium in order to act in the underlying target cells or even to reach systemic circulation, as well as overcome macrophagemediated phagocytosis in the alveolar space. Furthermore, neutrophils, together with the complement system, cytokines, chemokines, and other barriers to nanoparticles in the airways (12).

\section{Potentials of nanotechnology in asthma drug delivery}

\section{Traditional drugs improved by nanotechnology}

The authors discuss that the success of therapeutic strategies of asthmatic patients relies on adequate drug delivery to the lungs, thus achieving a high rate of airway anti-inflammatory efficacy while avoiding some of the side effects caused by prolonged glucocorticoid treatment. Thus, the study of inhaled nanoparticles depends upon critical evaluation of their capacity to effectively achieve high lung deposition. However, if the increased reached lung doses of drugs translates into better clinical efficacy with sustained therapeutic effects still needs to be further accessed $(13,14)$.

Nanoparticles have been manufactured from various materials_including polymers and metals—with unique architectures to serve as possible drug vehicles for particular diseases. The authors also note that technology for "nanomodification" of traditionally used anti-asthma drugs mostly focuses on research and development of distinct surface features nanoparticle carriers. Generally, material and particle size are selected to improve the stability of the aqueous suspension, with reduction of toxicity in mind $(15,16)$. Safety and efficacy have been demonstrated by appropriate clinical studies $(17,18)$.

In this context, few studies have been developing and testing new nanoparticles for asthma drug delivery. Telodendrimers, which are nontoxic nanocarriers were shown with better loading capacity and stability throughout the time (over 6 months) when compared to micelles. They allow slow delivery of formulations with hydrophobic substances, such as dexamethasone, directly to the lungs, mitigating airway allergic inflammation and hyperresponsiveness to a greater extent than same doses of carrier-free dexamethasone (19).

Patil-Gadhe and colleagues showed that a nanostructured lipid carrier loaded with montelukast bypassed hepatic metabolism, reducing hepatobiliary toxicity, and thus improving drug's therapeutic effects (20).

Thus, according to the literature, development of nanocarriers has already greatly improved the stability and performance of diverse drugs, and the development of novel nanoparticles focusing in improving drug delivery is an interesting and demanding clinical research field, particularly for new asthma therapies (21).

\section{Gene therapy formulations improved by nanotechnology}

Several asthmatic patients are refractory to most used therapies. For these patients, gene therapy has been shown to be an alternative, mainly focusing on inducing Th2 antagonists factors or inhibiting Th2 response. Under these circumstances, gene therapy has been conquering space as an alternative treatment for these patients and different structures for delivering a gene to a target organ, the nanoparticles, have been developed. However, our major obstacle that has been observed is to be able to develop structures for the delivery of biological materials that act differently depending on the organ to be treated and the desired sustained effects (22). Although easily accessible, the tracheobronchial tree remains a major challenge, because depending on the structure that will deliver the biological material, a greater stability property in physiological fluids is required to overcome barriers found in the lung $(23,24)$.

In order to overcome the physiological barriers of our target organ, the lung, different non-viral vectors have been developed based on different structures such as lipids and polymers aiming at a better pulmonary distribution of nanoparticles (12).

One of the most commonly used polycations is poly(ethylene imine) (PEI) for successful gene delivery, however this platform has been indicating high cytotoxicity (25). Different platforms for pulmonary delivery of biological materials, including polysaccharide chitosan (26), dendrimers (27), and poly(lactic-coglycolic acid) (PLGA)-based polymers (28) have also been described.

More recently, researchers developed a block copolymers of poly-L-lysine and polyethylene glycol linked by a cysteine residue (CK30PEG5K) for thymulin therapeutic delivery in an allergic asthma murine model, preventing important asthma hallmarks development, including collagen deposition and inflammation (29). This platform has been shown to be nonimmunogenic for human lungs in 
a phase I clinical trial (30).

Accordingly to different studies, the lack for vectors with high transfection potential and low immunogenicity is necessary not only for diseases of the respiratory system, but also for other organs. In this context, Mastorakos et al. demonstrated a biodegradable DNA nanoparticle able to overcome thick cystic fibrosis mucus barrier with no signs of lung cytotoxicity (22).

In conclusion, it is already known that asthma is an inflammatory pulmonary disorder with different cell and mediators involved that could be a possible molecular target for silencing or overexpression in the inflammatory cascade mediated by gene vectors. However, the safety and immunotoxicity need to be better investigated.

\section{Inorganic metallic nanoparticles}

Inorganic metallic nanoparticles, such as gold and silver, are widely used in chemistry and engineering as catalysts for reduction reactions. However, these materials have recently been explored in the biomedical field, mainly for cell and biomolecule labeling (Park et al., 2010). Due to their reducing potential, and capability to reduce reactive oxygen species (ROS), metallic nanoparticles can be used as antioxidants (Wong et al., 2009). Considering that the characteristic inflammation of asthma is the result of increased oxidative stress in the airways, gold (AuNP) and silver (AgNP) nanoparticles thus have therapeutic potential for asthma treatment.

Indeed, one study found that 6-nm AgNPs administered via nebulization $(20 \mathrm{ppm}, 40 \mathrm{mg} / \mathrm{kg}$ body weight/day) mitigated ROS generation, Th2-mediated inflammatory response, and airway resistance in a murine model (Park et al., 2010). Likewise, intranasally administered 6-nm AuNPs $(60 \mu \mathrm{g} / \mathrm{kg}$ animal weight $)$ minimized pivotal asthma symptoms in Swiss and $\mathrm{A} / \mathrm{J}$ mice, including airway hyperreactivity, inflammation, and pulmonary remodeling (Barreto et al, 2015). These beneficial effects resulted from suppression of the secretion of proinflammatory cytokines and chemokines, in a mechanism probably related to the downregulation of oxidative stress levels (Barreto et al., 2015). In both studies, the ovalbumin-induced allergic asthma model was used, and it is worth mentioning that metallic nanoparticles were administered prior to the OVA challenges.

Despite the potential of metallic nanoparticles, their safety in therapeutic airway procedures should be carefully examined prior to clinical use. Depending on the particle size and concentration, nanoparticles may increase the inflammatory response in asthmatic patients, just as natural micro- and nanoparticulate materials suspended in the environment do (Hussain et al., 2011); this has been observed in some preclinical studies. After exposure of control and allergic BALB/C mice to 33-nm AgNPs, increased protein production in plasma and bronchoalveolar lavage was observed. These proteins are involved in processes such as blood coagulation, plasminogen activation, T-lymphocyte activation, and cytokine and chemokine production, suggesting that AgNPs modulate immune processes (Su et al., 2013). Likewise, after intratracheal inoculation of AuNPs $(0.8 \mathrm{mg} / \mathrm{kg})$, airway hyperreactivity and inflammatory responses were worsened in BALB/c mice (Hussain et al., 2011).

\section{Nanotechnology in the stem-cell field}

Therapies based on stem cells have been extensively investigated in experimental and clinical trials as an innovative therapeutic approach for lung diseases, due to their anti-inflammatory and reparative effects. In this context, stem cells are known to act mainly through the secretion of endocrine or paracrine mediators, including extracellular vesicles (EVs). EVs are intercellular communication mediators, as they carry microRNA, mRNA, proteins, and lipid mediators that can induce beneficial effects similar to those of source cells. EVs, exosomes (50-200 nm), and microvesicles (150-1,000 nm) from mesenchymal stromal cells have all been tested in animal models of allergic airway disease. EVs obtained from bone marrow-derived mesenchymal stromal cells improved lung mechanics, reduced peribronchial inflammation, and mitigated the Th2 and Th17 cell responses (Cruz et al., 2015). EVs from adipose tissue-derived mesenchymal stromal cells were also able to reduce inflammation and airway remodeling in an experimental asthma model (de Castro et al., 2017).

\section{Summary and future directions}

Nanotherapy has been investigated as a promising strategy to treat asthma. Experimental studies have shown that nanocarriers are safe and effective. Although much preclinical research has focused on nanotechnology as a potential alternative for the treatment of asthma, surprisingly, no clinical study has been done to date. Many challenges still need to be overcome before nanotechnology 
can be cross from bench to bedside. A better comprehension of their mechanisms of action and nanocarriers chemical structures are crucial for the implementation of more reasonable trials.

\section{Acknowledgments}

The authors thank Mr. Filippe Vasconcellos (São Paulo), Brazil, for his assistance in editing the manuscript.

Funding: This study was supported by the Brazilian Council for Scientific and Technological Development (CNPq), the Rio de Janeiro State Research Foundation (FAPERJ), and the Coordination for the Improvement of Higher Education Personnel (CAPES).

\section{Footnote}

Conflicts of Interest: The authors have no conflicts of interest to declare.

Ethical Statement: The authors are accountable for all aspects of the work in ensuring that questions related to the accuracy or integrity of any part of the work are appropriately investigated and resolved.

Open Access Statement: This is an Open Access article distributed in accordance with the Creative Commons Attribution-NonCommercial-NoDerivs 4.0 International License (CC BY-NC-ND 4.0), which permits the noncommercial replication and distribution of the article with the strict proviso that no changes or edits are made and the original work is properly cited (including links to both the formal publication through the relevant DOI and the license). See: https://creativecommons.org/licenses/by-nc-nd/4.0/.

\section{References}

1. GINA. Global Strategy for Asthma Management and Prevention 2019. Available online: https://ginasthma.org/ wp-content/uploads/2019/06/GINA-2019-main-reportJune-2019-wms.pdf

2. Fernandes SSC, Andrade CR, Alvim CG, et al. Epidemiological trends of allergic diseases in adolescents. J Bras Pneumol 2017;43:368-72.

3. Andersson CK, Weitoft M, Rydell-Tormanen K, et al. Uncontrolled asthmatics have increased FceRI(+) and TGF-beta-positive MCTC mast cells and collagen VI in the alveolar parenchyma. Clin Exp Allergy
2018;48:266-77.

4. Usmani OS, Lavorini F, Marshall J, et al. Critical inhaler errors in asthma and COPD: a systematic review of impact on health outcomes. Respir Res 2018;19:10.

5. Chung KF. New treatments for severe treatment-resistant asthma: targeting the right patient. Lancet Respir Med 2013;1:639-52.

6. Papierniak ES, Lowenthal DT, Harman E. Novel therapies in asthma: leukotriene antagonists, biologic agents, and beyond. Am J Ther 2013;20:79-103.

7. Anandan C, Nurmatov U, van Schayck OC, et al. Is the prevalence of asthma declining? Systematic review of epidemiological studies. Allergy 2010;65:152-67.

8. Omlor AJ, Nguyen J, Bals R, et al. Nanotechnology in respiratory medicine. Respir Res 2015;16:64.

9. da Silva AL, Cruz FF, Rocco PRM, et al. Correction to: New perspectives in nanotherapeutics for chronic respiratory diseases. Biophys Rev 2017;9:805.

10. Kim N, Duncan GA, Hanes J, et al. Barriers to inhaled gene therapy of obstructive lung diseases: A review. J Control Release 2016;240:465-88.

11. Schneider CS, Xu Q, Boylan NJ, et al. Nanoparticles that do not adhere to mucus provide uniform and long-lasting drug delivery to airways following inhalation. Sci Adv 2017;3:e1601556.

12. Di Gioia S, Trapani A, Castellani S, et al. Nanocomplexes for gene therapy of respiratory diseases: Targeting and overcoming the mucus barrier. Pulm Pharmacol Ther 2015;34:8-24.

13. Franzese C. Management of acute asthma exacerbations. Int Forum Allergy Rhinol 2015;5 Suppl 1:S51-6.

14. Wang K, Feng Y, Li S, et al. Oral Delivery of BavachininLoaded PEG-PLGA Nanoparticles for Asthma Treatment in a Murine Model. J Biomed Nanotechnol 2018;14:1806-15.

15. Da Silva AL, Santos RS, Xisto DG, et al. Nanoparticlebased therapy for respiratory diseases. An Acad Bras Cienc 2013;85:137-46.

16. Chandel A, Goyal AK, Ghosh G, et al. Recent advances in aerosolised drug delivery. Biomed Pharmacother 2019;112:108601.

17. Zhang L, Gu FX, Chan JM, et al. Nanoparticles in medicine: therapeutic applications and developments. Clin Pharmacol Ther 2008;83:761-9.

18. Weers J, Metzheiser B, Taylor G, et al. A gamma scintigraphy study to investigate lung deposition and clearance of inhaled amikacin-loaded liposomes in healthy male volunteers. J Aerosol Med Pulm Drug Deliv 2009;22:131-8. 
19. Kenyon NJ, Bratt JM, Lee J, et al. Self-assembling nanoparticles containing dexamethasone as a novel therapy in allergic airways inflammation. PLoS One 2013;8:e77730.

20. Patil-Gadhe A, Kyadarkunte A, Patole M, et al. Montelukast-loaded nanostructured lipid carriers: part II pulmonary drug delivery and in vitro-in vivo aerosol performance. Eur J Pharm Biopharm 2014;88:169-77.

21. Renner N, Steckel H, Urbanetz N, et al. Nano- and Microstructured model carrier surfaces to alter dry powder inhaler performance. Int J Pharm 2017;518:20-8.

22. Mastorakos P, da Silva AL, Chisholm J, et al. Highly compacted biodegradable DNA nanoparticles capable of overcoming the mucus barrier for inhaled lung gene therapy. Proc Natl Acad Sci U S A 2015;112:8720-5.

23. Sanders N, Rudolph C, Braeckmans K, et al. Extracellular barriers in respiratory gene therapy. Adv Drug Deliv Rev 2009;61:115-27.

24. Isidoro-Garcia M, Sanchez-Martin A, Garcia-Sanchez A, et al. Pharmacogenetics and the treatment of asthma. Pharmacogenomics 2017;18:1271-80.

25. Beyerle A, Irmler M, Beckers J, et al. Toxicity pathway focused gene expression profiling of PEI-based polymers

Cite this article as: da Silva AL, Silva LA, Cruz FF, Rocco PR, Morales MM. Application of novel nanotechnologies in asthma. Ann Transl Med 2020;8(5):159. doi: 10.21037/atm.2019.12.40 for pulmonary applications. Mol Pharm 2010;7:727-37.

26. Koping-Hoggard M, Tubulekas I, Guan H, et al.

Chitosan as a nonviral gene delivery system. Structureproperty relationships and characteristics compared with polyethylenimine in vitro and after lung administration in vivo. Gene Ther 2001;8:1108-21.

27. Rudolph C, Lausier J, Naundorf S, et al. In vivo gene delivery to the lung using polyethylenimine and fractured polyamidoamine dendrimers. J Gene Med 2000;2:269-78.

28. Bivas-Benita M, Lin MY, Bal SM, et al. Pulmonary delivery of DNA encoding Mycobacterium tuberculosis latency antigen Rv1733c associated to PLGA-PEI nanoparticles enhances T cell responses in a DNA prime/protein boost vaccination regimen in mice. Vaccine 2009;27:4010-7.

29. da Silva AL, Martini SV, Abreu SC, et al. DNA nanoparticle-mediated thymulin gene therapy prevents airway remodeling in experimental allergic asthma. J Control Release 2014;180:125-33.

30. Konstan MW, Davis PB, Wagener JS, et al. Compacted DNA nanoparticles administered to the nasal mucosa of cystic fibrosis subjects are safe and demonstrate partial to complete cystic fibrosis transmembrane regulator reconstitution. Hum Gene Ther 2004;15:1255-69. 